\title{
FRET-based tension measurement across actin-associated mechanotransductive structures using Lima1
}

\author{
SAYUKI HIRANO, TAKAYOSHI YAMAMOTO and TATSUO MICHIUE* \\ Department of Life Sciences (Biology), Graduate School of Arts and Sciences, The University of Tokyo, Tokyo, Japan
}

\begin{abstract}
During embryogenesis, mechanical forces play important roles in morphogenesis and tissue differentiation. To measure these forces, we developed a new Förster resonance energy transfer (FRET)-based tension sensor that uses the actin-associated protein, Lima1. The sensor was validated in HeLa cells where we found the FRET index decreased or increased in response to changes in the cellular environment. Introducing the sensor into Xenopus embryos enabled us to observe tension distributions and sequential changes over the entire embryo. We found that during neural tube closure, neural ectodermal cells showed a gradual decrease in their FRET index, indicating they generate higher tension with the progression of neural tube closure. With appropriate controls and application, our Lima1 tension sensor has the ability to uncover and define other tension distributions and their functions in development and differentiation.
\end{abstract}

KEY WORDS: Lima1, FRET, tension sensor, actin filament, Xenopus

\section{Introduction}

Morphogenic processes, such as invagination, tube formation, epiboly, and convergent extension, are widespread during early embryonic development. During morphogenesis cells can generate and sense various mechanical forces (Desprat et al., 2008; Heer and Martin, 2017; West et al., 2017), and there are reports indicating strain-force and other mechanical forces can influence the behavior and differentiation of a cell (Engler et al., 2006; Geuss et al., 2014; He et al., 2018; Kumar et al., 2017). Thus, a better understanding of mechanical cues in development is becoming increasingly important.

Various methods can be used to measure mechanical forces in organisms, including the recently developed Förster resonance energy transfer (FRET)-based tension measurement technique with associated FRET-based tension sensors (Grashoff et al., 2010; Kumar et al., 2016; Meng and Sachs, 2011; Yamashita et al., 2016). A FRET-based tension sensor (TS) module consists of a pair of fluorescent proteins linked by an elastic linker. Inserting the module into a cell adhesion molecule allows the tension across cell-cell or cell-ECM interfaces to be observed. In contrast to methods such as laser ablation, FRET-based tension sensors does not disrupt tissue structure and can therefore be used to broadly and sequentially measure tension in vivo (Krieg et al., 2014; Lagendijk et al., 2017; Yamashita et al., 2016).
We developed a new FRET-based tension sensor using the cytoskeleton-associated protein, Lima1, also known as EPLIN. Lima1 belongs to the LIM family, and has two actin-binding sites in the $\mathrm{N}$ - and $\mathrm{C}$-terminal domains. Through these domains it associates with the cadherin-catenin complex and actin filaments (Abe and Takeichi, 2008; Maul et al., 2003). Lima1 is localized along the zonula adherens in epithelial and endothelial cell monolayers, while mainly localized along actin stress fibers in sparse cells (Chervin-Pétinot et al., 2012). Thus, the Lima1 tension sensor, FRET module inserted Lima1, should be able to measure tension over various actin-based mechanotransducive structures.

In this study, we constructed a Lima1 tension sensor, validated it in cultured cells, then introduced the sensor into Xenopus embryos to show it could sequentially and noninvasively measure cellular tension.

\section{Experimental Protocols}

\section{Constructs}

We used our previously published tension sensor module (Yamashita et al., 2016), which consists of EGFP and mCherry linked

Abbreviations used in this paper: FRAP, fluorescence recovery after photobleaching; FRET, Förster resonance energy transfer.

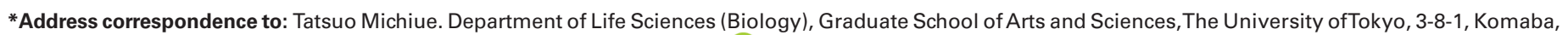
Meguro-ku,Tokyo 153-8902 Japan. E-mail: tmichiue@bio.c.u-tokyo.ac.jp - (iD https://orcid.org/0000-0001-9047-0513
}

Submitted: 27 March, 2018; Accepted: 31 May, 2018.

Supplementary Material (two figures) for this paper is available at: https://doi.org/10.1387/ijdb.180110tm

ISSN: Online 1696-3547, Print 0214-6282 
by an elastic linker sequence (GPGGA) ${ }_{8}$ derived from spider silk protein, flagelliform. To construct LimaTS, the module was inserted into Xenopus Lima1 immediately after the $\mathrm{N}$-terminal actin-binding domain, between Ala355 and Arg356. For LimaTS-EGFP (-) or LimaTS-mCherry (-) (fluorescent mutants of LimaTS), Tyr66 of the EGFP domain or Tyr72 of the mCherry domain were each replaced with a leucine. To construct LimaCS and Lima1-mCherry, the tension sensor module and mCherry were ligated to the Cterminus of Lima1.

\section{Cell culture conditions and transfection}

HeLa cells were cultured in high glucose DMEM (Wako, Osaka, Japan) containing 10\% fetal bovine serum (Thermo Fisher Scientific, Tokyo, Japan) and 1\% penicillin-streptomycin (SIGMA, Kanagawa, Japan) at $37^{\circ} \mathrm{C}$ in $5 \% \mathrm{CO}_{2}$. Cells were seeded in 4-well coverglass chambers (IWAKI, Shizuoka, Japan) and transfected using Lipofectamine 3000 transfection kit (Thermo Fisher Scientific) in accordance with the manufacturer's guidelines. Cells were analyzed after an overnight incubation. To stretch stress fibers, cells were incubated in 30\% milliQ water/DMEM for $5 \mathrm{~min}$. To inhibit actomyosin constriction, cells were incubated in $5 \mu \mathrm{M}$ Cytochalasin B (Wako)/0.025\% DMSO/DMEM or 10 $\mathrm{MM}$ Y-27632 (ADooQ, CA, USA)/0.002\%DMSO/DMEM for 1 hour. For the control condition of Cytochalasin B treatment, cells were incubated in $0.025 \%$ DMSO/ DMEM. As the Y-27632 solution contained such a low concentration of DMSO, the control condition for Y-27632 treatment was DMEM with no DMSO.

\section{Phalloidin staining}

Cells expressing LimaTS-EGFP (-) were fixed in $4 \%$ paraformaldehyde/PBS for 10 min and permeabilized with $0.5 \%$ Triton X-100/ PBS for $5 \mathrm{~min}$. After PBS washing, cells were stained withAlexa Fluor 488 phalloidin (Thermo Fisher Scientific) for $30 \mathrm{~min}$ and washed with PBS. For embryos, vitelline membranes were removed, and embryos were fixed in MEMFA (0.1M MOPS, 2mM EGTA, $1 \mathrm{mM}$ $\mathrm{MgSO}_{4}, 3.7 \%$ formaldehyde) for 1 hour. After permeabilization in PTw (0.1\% Tween20/PBS) for $30 \mathrm{~min}$, embryos were stained with Alexa Fluor 488 phalloidin for $20 \mathrm{~min}$, and then washed with PBS. All processes were conducted at room temperature in the dark.

\section{Embryo culture and microinjection}

Xenopus laevis embryos were obtained via artificial fertilization and cultured in $1 \times$ Steinberg's solution. At the 4-cell stage, mRNA was injected intothe animal marginal zone in $5 \%$ Ficoll $/ 1 \times$ Steinberg's solution. Injection doses were 3.5ng of LimaTS, 1.25ng of LimaCS, 3.5ng of LimaTS-EGFP (-), and 3.5ng of LimaTS-mCherry (-). To define the midline of the embryo, Alexa Fluor 647 was injected into one side of the dorsal blastomeres. After incubation in Ficoll solution to the appropriate stage, embryos were washed with $1 \times$ Steinberg's solution and used for imaging.

\section{Confocal imaging}

All images in this study were acquired with a confocal microscope (Olympus, Tokyo, Japan). An UPLSAPO40XS silicon immersion objective was used for imaging HeLa cells and an UPLSAPO10X2 objective was used for embryos. For embryo imaging, a $35 \mathrm{~mm} /$ glass-based dish (IWAKI) was used with embryos embedded in 1.2\% low melting point Agarose (Thermo Fisher Science) $1 \times$ Steinberg's solution.

\section{FRET calculation}

FRET index was calculated with a custom script in ImageJ software using the same calculation algorithm as we have used previously (Yamashita et al., 2016). The first step of calculation was subtracting the acceptor bleed-through (ABT) and donor bleed-through (DBT) from the raw FRET. The ABT and DBT were determined by multiplying acceptor and donor intensity by the bleed-through coefficients. The bleed-through corrected FRET was then divided by the acceptor intensity to normalize for the amount of sensor protein. The pixel-by-pixel correction of experimental data for bleed-through and the amount of protein was performed according to the following equation:

$$
\text { FRET index }=\frac{F-c_{a} A-c_{d} q D}{A}
$$

where $F=$ raw FRET, $A=$ acceptor intensity, and $q D=$ quenched donor intensity, $c a=$ acceptor bleed-through coefficient, $c d=$ donor bleed-through coefficient. Acceptor bleed-through coefficient was determined using cells or embryos expressing LimaTS-EGFP (-). The raw FRET was divided by the acceptor intensity and used as ca. Donor bleed-through coefficient was determined in the analogous way using LimaTS-mCherry (-).

\section{Quantification of FRET index}

For cells, the outline of the cell was determined manually, and the average value of that region was used as the cell's FRET index. For embryos, the FRET index along cell membranes was determined using the original fluorescence images. An "edge" was defined as a line from one intersection of cell membranes to another, and the positional information of the edge was determined manually. From this positional information, the FRET index of the edge was measured, and the average value along the edge was used as the edge's FRET index, with 40-100 edges quantified per embryo image. In this study, the "edge" included both ends of vertexes.

\section{FRAP analysis}

Five prebleach images were acquired and then a laser pulse at $100 \%$ power of the $559 \mathrm{~nm}$ line was used to bleach ROI. ROI was selected from regions showing well-developed stress fibers. Timelapse images were acquired every $5 \mathrm{~s}$ for $250 \mathrm{~s}$. Average intensity of ROI was calculated and then normalized with the intensity of base ROI and whole cell ROI (the double normalization).

\section{FRET analysis with stretched animal cap cells}

Each mRNA was injected into Xenopus embryos in the same way as Figs. 3, 4. Animal caps (LimaTS: $n=2$, LimaCS: $n=2$ ) were dissected at St.9 and attached to silicon stretch chambers (STREX, Osaka, Japan) which had been coated with fibronectin at $37^{\circ} \mathrm{C}$ overnight in advance. After incubation for 1 hour at $24^{\circ} \mathrm{C}$, animal caps were stretched by $10 \%$. The FRET indices along cell membranes were determined in the same way as Figs. 3, 4.

\section{Results}

\section{Construction and subcellular characterization of the Lima1 tension sensor}

We previously developed a FRET-based tension sensor to visualize tension distribution in whole embryos (Yamashita et 
al., 2016). Here we used the same tension sensor (TS) module, which consists of an EGFP (donor) and an mCherry (acceptor) linked by an elastic linker peptide derived from spider silk protein, flagelliform (Fig. 1A). Since FRET is sensitive to the distance between fluorescent molecules, FRET efficiency should decrease in concordance with the force stretching the module (Fig. 1B). Lima1 interacts with actin filaments and the cadherin-catenin complex via its $\mathrm{N}$ - and C-terminal domains to connect these components (Abe and Takeichi, 2008; Maul et al., 2003). As such, the module was inserted between the head actin-binding domain and LIM domain, and was named LimaTS (Fig. 1C). LimaCS (control sensor), which exhibits constant FRET, was made by attaching the TS module to the C-terminus of Lima1 (Fig. 1D).

To confirm the localization of LimaTS, HeLa cells were transfected with LimaTS-EGFP (-) (a fluorescent mutant of LimaTS) and stained for actin filaments (Fig. 1E). LimaTS-EGFP (-) co-localized with actin stress fibers, with some also present in the cytoplasm. Furthermore, to test the dynamics of LimaTS, we performed a FRAP (fluorescence recovery after photobleaching) analysis (Fig. 1F). When mCherry was photobleached in a small region inside cells, LimaTS showed similar recovery rates to mCherry-tagged Lima1. These data show that insertion of the TS module does not significantly affect the intracellular localization and dynamics of Lima1.

\section{FRET calculation and validation of the tension sensor}

To confirm the function of LimaTS and LimaCS, we transfected these sensors into HeLa cells and calculated the FRET index. The first step of calculation was subtracting the acceptor bleed-through $(\mathrm{ABT})$ and donor bleed-through (DBT) from the raw FRET. The bleed-through corrected FRET was then divided by the acceptor intensity to normalize for the amount of sensor protein. The calculated FRET index was displayed in a heat map (Fig. 2A).

We validated the sensor function by changing the tension being applied to stress fibers in living cells. First, we incubated cells expressing LimaTS or LimaCS in hypotonic medium where cells will swell, and tension should increase (Fig. 2 B-D). The FRET index of LimaTS but not LimaCS decreased in response to the increase in tension. Next, to inhibit actomyosin function, we treated cells with Cytochalasin B or Y-27632 (Fig. 2 E-J, Supplementary Fig. 1 C-F). We confirmed that Cytochalasin B- and Y-27632-treated cells lost filament-like fluorescence distribution, indicating that the stress fibers were disrupted (data not shown). Under such low-tension conditions, LimaTS but not LimaCS showed a higher FRET index. We could also observe the change of FRET index by stretching animal cap cells (Fig. S3). These results indicate that LimaTS can detect tension change, while LimaCS maintains a constant FRET.

\section{Introducing the tension sensor into Xenopus embryos}

Next, we introduced the tension sensors into Xenopus embryos and measured tension in ectodermal tissue at an early developmental stage. In ectodermal cells, cadherins and actin filaments are localized along cell membranes and form the zonula adherens (Nandadasa et al., 2009). Introducing Lima1-mCherry into the embryos revealed that Lima1 was co-localized with circumferential actin filaments (Fig. 3A). We then injected LimaTS and LimaCS mRNAinto embryos and confirmed that both sensors were normally localized along cell membranes (Fig. 3 B,C insets). We also confirmed that mRNA-injected embryos showed normal phenotypes (Supplementary Fig. 2). The FRET indices were calculated and quantified (Fig. 3 B-E). Some sensor protein was observed in the
A

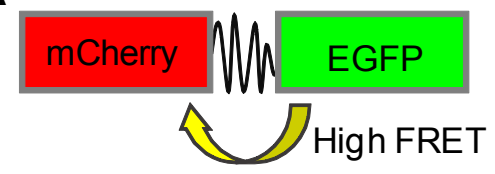

B

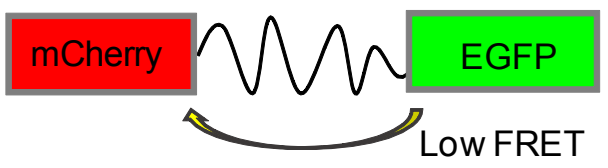

E

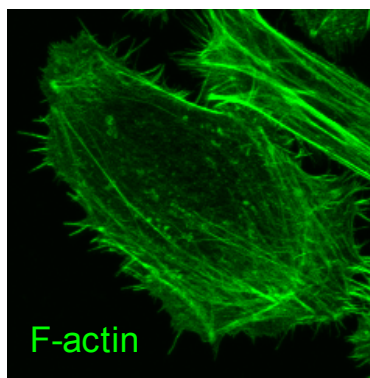

C LimaTS

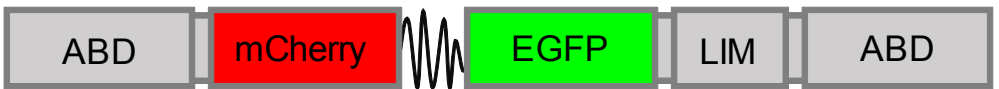

D LimaCS
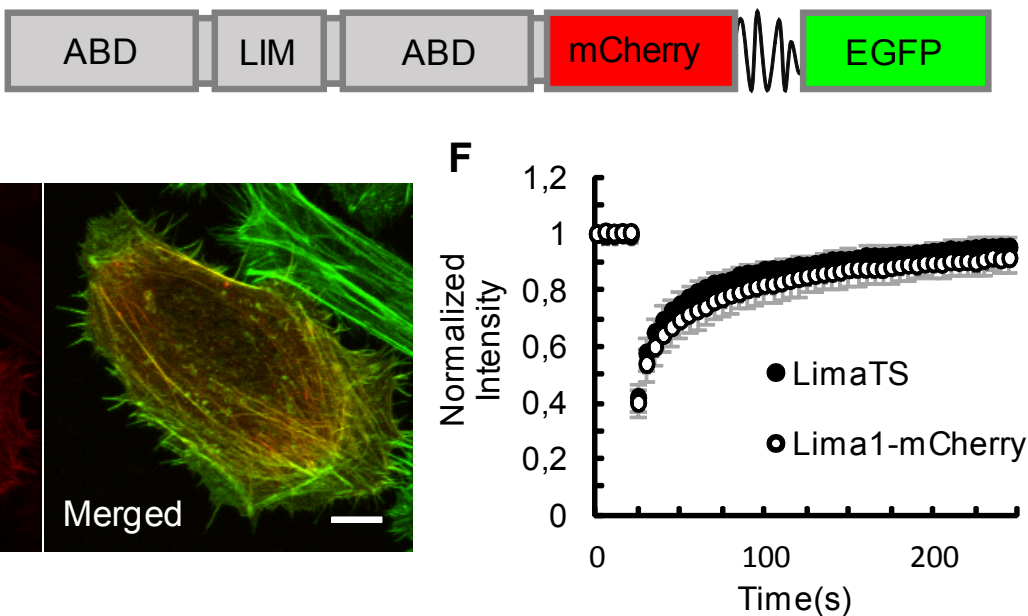

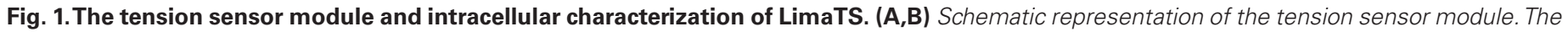

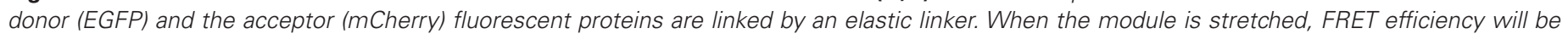

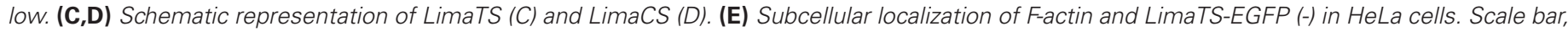
$10 \mu \mathrm{m}$. (F) Fluorescence recovery rates of LimaTS $(n=19)$ and Lima1-mCherry $(n=12)$. Error bars represent standard error of the mean. 
cytoplasm, where it would not reflect the tension generated on the junctions. The FRET index was determined along cell membranes using the original fluorescence images (Fig. 3D). An "edge" was defined as a line from one intersection of cell membranes to another. From this positional information, the FRET index of the edge was measured, and the average value along the edge was used as the edge's FRET index. In this way, we quantified FRET index on the ectodermal cell-cell junctions over the whole embryo and found that LimaTS had a lower index value than LimaCS (Fig. 3E). Considering that LimaTS showed higher FRET index than LimaCS in cultured cells with no treatment (Fig. 2), this result suggests that ectodermal cells are under a certain level of stress along the entire embryo.

\section{Tension change during neural tube closure}

In neural tube closure (NTC), neural plate cells generally reduce their apical surface area (apical constriction), contributing to the bending of neural folds (Nikolopoulou et al., 2017). Actomyosin dynamics and resulting mechanical forces appear to be important in promoting this morphogenesis (Escuin et al., 2015; Rolo et al., 2009). Therefore, we measured tension change in the neural plate during NTC. Comparison between St.13 (before the beginning of NTC) and St.18 (immediately before the completion of NTC) of LimaTS-expressing embryos revealed that the FRET index decreased as NTC progressed (Fig. 4 A,B). Quantification of the FRET index along cell membranes in a time course found the FRET index of LimaTS gradually decreased during NTC, while LimaCS did not (Fig. 4C). This indicates that neural plate cells gradually develop higher tension along their zonula adherens concurrently with the progression of NTC.

\section{Discussion}

In this study, we demonstrated our tension sensor was useful for measuring tension in living cells and tissues. Tension could be visualized in embryos broadly, sequentially and noninvasively, which was similar to our previously published tension sensor (Yamashita et al., 2016). In contrast with our previous sensor, however, LimaTS

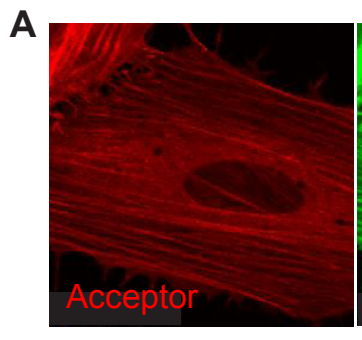

B

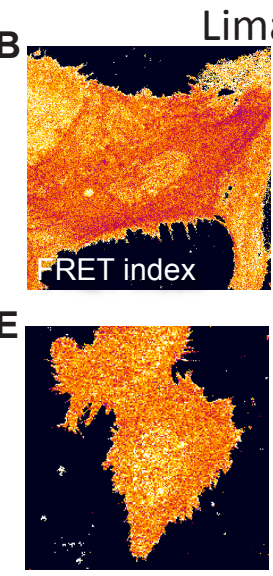

H
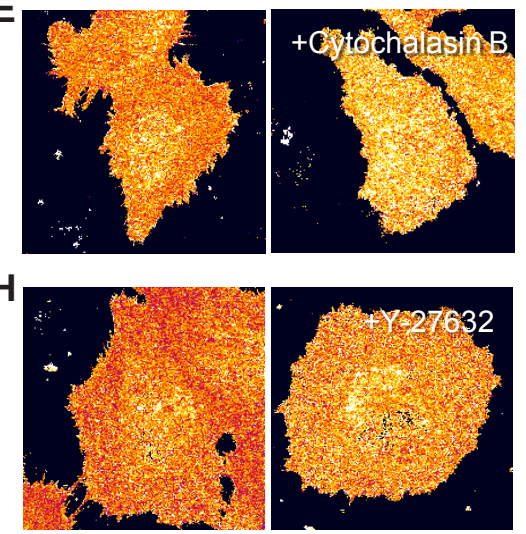
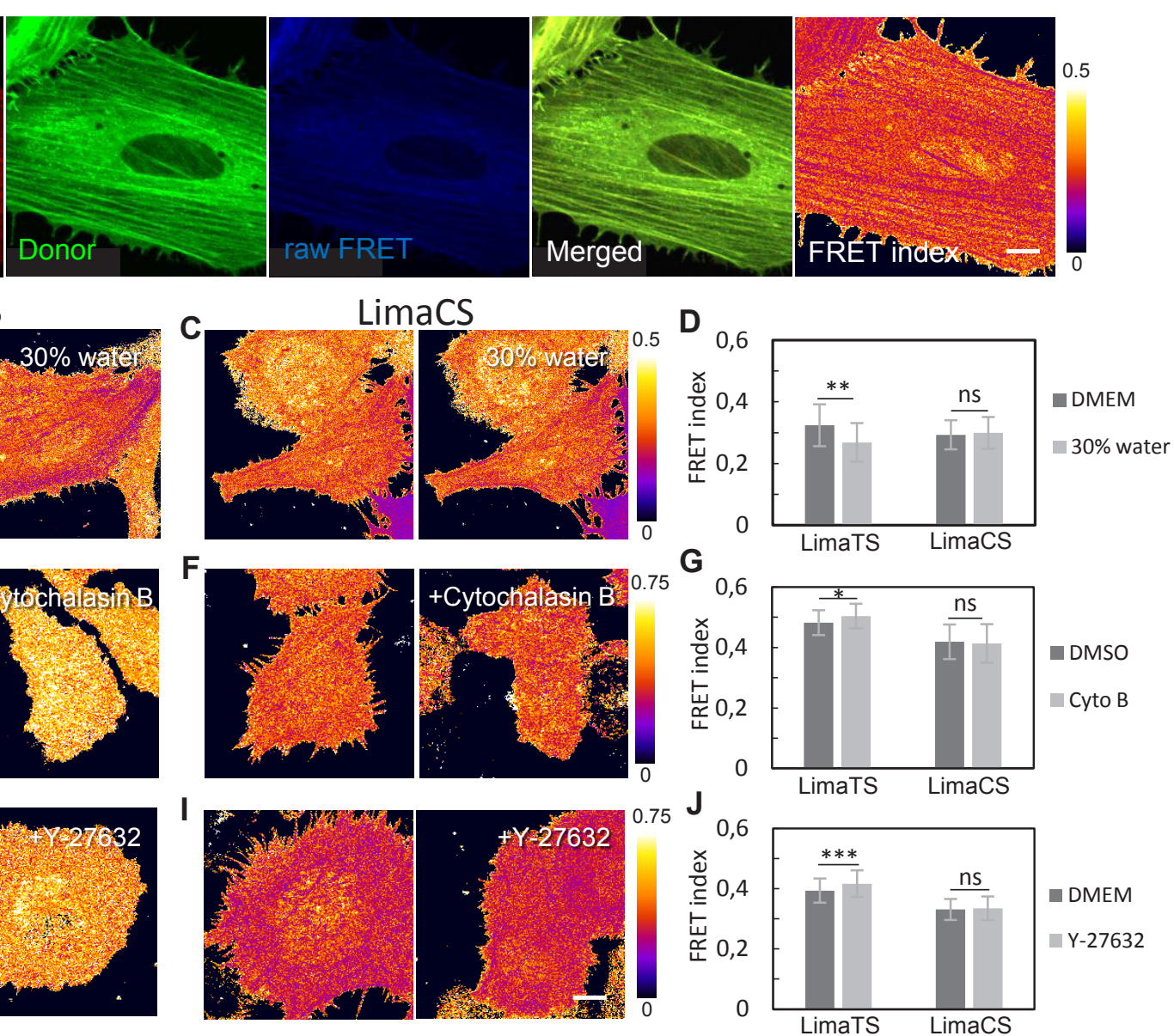

Fig. 2. Förster resonance energy transfer (FRET) calculation and validation of Lima1 tension sensor. (A) Representative images of HeLa cells transfected with LimaTS. Acceptor, donor, and raw FRET images are represented as red, green, and blue channels, respectively. The FRET index is calculated from these images and displayed in a heat map. Scale bar, $10 \mu \mathrm{m}$. (B,C) FRET index images of cells expressing LimaTS (B) or LimaCS (C) before and after changing medium from DMEM to 30\% water/DMEM. (D) Quantified FRET index of LimaTS (n=21) and LimaCS ( $n=20)$. ** $\mathrm{P}<0.01$. (E,F) Cells expressing LimaTS (E) or LimaCS (F) incubated in 0.025\% DMSO/DMEM or 5 uM Cytochalasin B/0.025\% DMSO/DMEM. (G) Quantified FRET index of LimaTS (DMSO: $n=51$, Cytochalasin B: $n=44)$ and LimaCS (DMSO: $n=50$, Cytochalasin B: $n=49) .{ }^{*} \mathrm{P}<0.05$. (H,I) Cells expressing LimaTS (H) or LimaCS (I) incubated in DMEM or $10 \mu M$ Y-27632/DMEM. Scale bar, $10 \mu \mathrm{m}$. (J) Quantified FRET index of LimaTS (DMEM: $n=64, Y-27632: n=65)$ and LimaCS (DMEM: $n=80, Y-27632: n=72)$. ${ }^{* *} \mathrm{P}<0.001$. All error bars represent the standard error of the mean. 


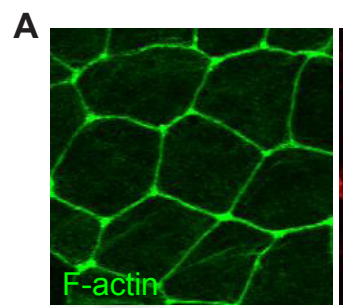

B

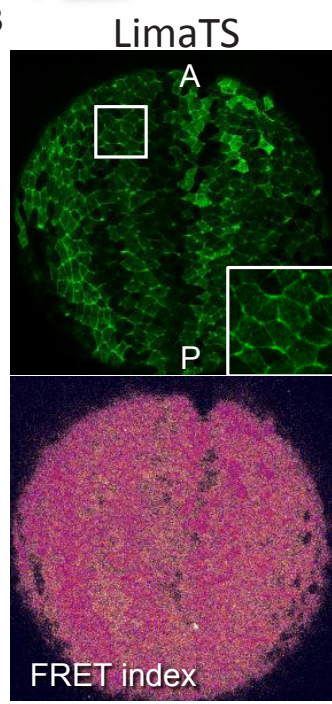

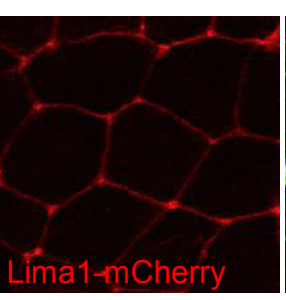

C

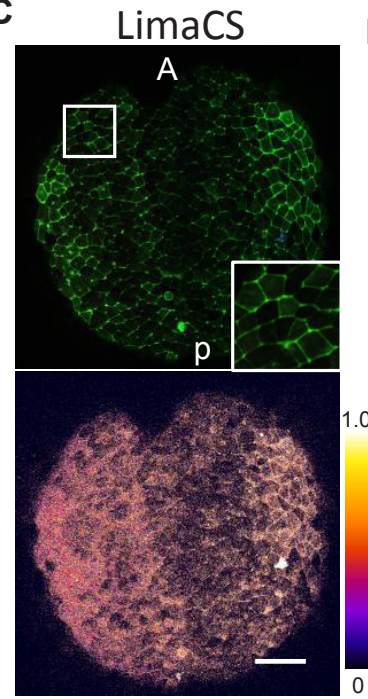

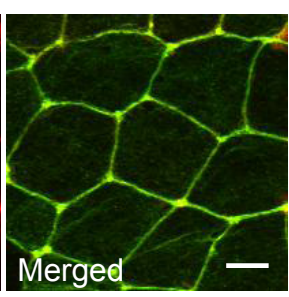

$\mathrm{D}$

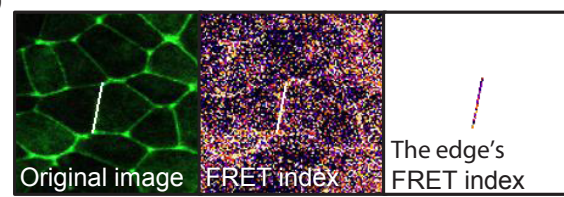

$\mathrm{E}$

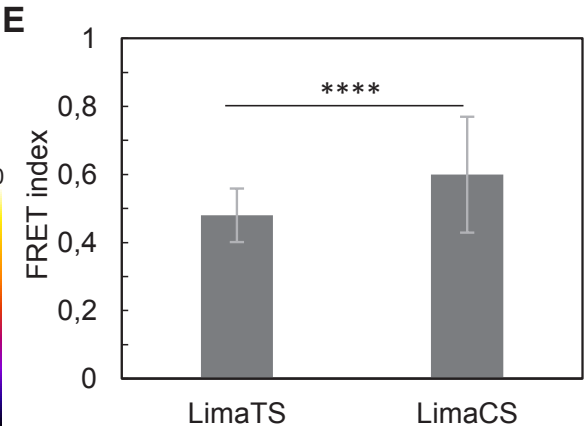

Fig. 3. Lima1 localization and tension measurement in Xenopus embryos. (A) Subcellular localization of F-actin and Lima 1-mCherry in Xenopus ectodermal tissue. Scale bar, 10 um. (B,C) Dorsal view images of St. 13 embryos expressing LimaTS (B) or LimaCS (C). Insets are magnifications of the boxed areas of the whole embryos. EGFP and mCherry images are merged (upper) and FRET index is displayed in heatmap (lower). A: Anterior, P: Posterior. Scale bar, $200 \mu \mathrm{m}$. (D) An example of defining an edge and measuring the edge's FRET index. (E) Quantified FRET index of LimaTS $(n=5)$ and LimaCS $(n=4)$. **** $\mathrm{P}<0.0001$. Error bars represent the standard error of the mean.
A

LimaTS
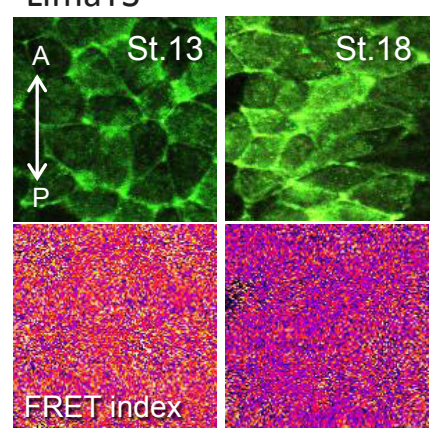

B LimaCS

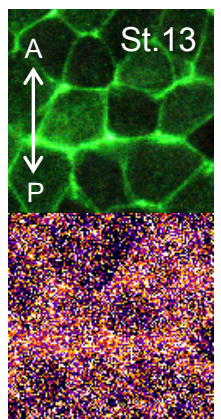

C

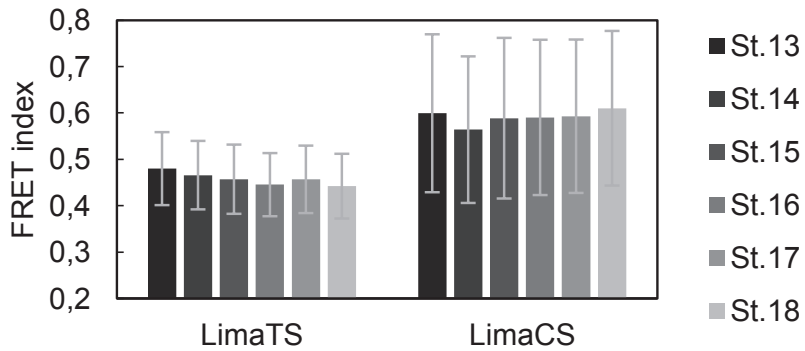

Fig. 4. Tension changes during neural tube closure. (A,B) Neural ectoderm of embryos expressing LimaTS (A) or LimaCS (B) at St.13 (before the beginning of neural tube closure) and St.18 (immediately before the completion of neural tube closure). EGFP and mCherry images are merged (upper) and FRET index is displayed in heatmap (lower). Scale bar, $100 \mu \mathrm{m}$. (C) Stage time course of FRET index during neural tube closure. Images of embryos expressing LimaTS ( $n=5)$ or LimaCS $(n=4)$ were taken for each stage, and the FRET index of the neural plate was quantified. Error bars represent the standard error of the mean. showed a higher FRET index than LimaCS in untreated cultured cells. This may be because TS module of LimaTS is more compacted between Lima1 subunits, compared with that of LimaCS in stress-free conditions. Another possibility is that the angle of the module in LimaTS is formed to be suitable for energy transfer. But this relationship was reversed in embryos. Ectodermal tissues should generally be under certain tension, thus agreeing with our observation of the FRET index of LimaTS being lower than that of LimaCS. The FRET index of LimaTS was lower still in the neural plate, with the progression of NTC. Actomyosin in neural plate cells are considered to generate higher tension as NTC progresses, contributing to the bending of neural folds (Sawyer et al., 2010).

The acceptor fluorescence intensity and FRET index were negatively correlated in cells and embryos. While the negative correlations were weak, they should not be ignored when evaluating differences in tension. If the acceptor intensity distributions are significantly different between two samples, the resulting difference in FRET index may be spurious. To avoid possible misinterpretation, we ensured the samples being compared did not show any significant difference in acceptor intensity distributions. While this confirmation does not indicate perfect correspondence in acceptor intensity distributions, it does support the validity of our findings using our sensors.

FRET-based tension sensor approaches can be complex, with many factors to consider. These include ensuring the sensor proteins show normal intracellular characteristics and their expression level is controlled so as not to disrupt normal development. These complexities, however, are 
outweighed by the many events we can investigate with this sensor. For example, in the neural plate, the accumulation of pMLC (phosphorylated myosin light chain) and rates of junction shrinkage differ depending on the junction angle (Christodoulou and Skourides, 2015; Nishimura et al., 2012). Distinguishing each cell-cell interface as we did here, would provide a more detailed understanding of how these factors correlate. Additionally, the sensor detects tension along the ectodermal zonula adherens as well as other actin-based mechanosensitive structures such as stress fibers, which greatly extends the possible utilities of this sensor. When applied appropriately, FRET sensors will help us reveal and precisely delineate the presence of mechanical forces and their function in developing and differentiating tissues.

\section{Acknowledgement}

We thank Prof. Takashi Tsuboi and Prof. Tetsuya Kitaguchi and Dr. Satoshi Yamashita for helpful advices. This work was supported in part by a funding from Grants-in-Aid for Scientific Research [grant no. 26440115, 16KT0065 and 18H04967] and Nakatani foundation for TM.

\section{References}

ABE K, TAKEICHI M (2008). EPLIN mediates linkage of the cadherin catenin complex to F-actin and stabilizes the circumferential actin belt. Proc Natl Acad Sci USA 105: 13-19.

CHERVIN-PÉTINOT A, COURÇON M, ALMAGRO S, NICOLAS A, GRICHINE A, GRUNWALD D, PRANDINI MH, HUBER P, GULINO-DEBRAC D (2012). Epithelial Protein Lost In Neoplasm (EPLIN) interacts with $\alpha$-catenin and actin filaments in endothelial cells and stabilizes vascular capillary network in vitro. $J$ Biol Chem 287: 7556-7572.

CHRISTODOULOU N, SKOURIDES PAA (2015). Cell-Autonomous Ca2+Flashes Elicit Pulsed Contractions of an Apical Actin Network to Drive Apical Constriction during Neural Tube Closure. Cell Rep 13: 2189-2202.

DESPRAT N, SUPATTO W, POUILLE PA, BEAUREPAIRE E, FARGE E (2008). Tissue Deformation Modulates Twist Expression to Determine Anterior Midgut Differentiation in Drosophila Embryos. Dev Cell 15: 470-477.

ENGLER AJ, SEN S, SWEENEY HL, DISCHER DE (2006). Matrix Elasticity Directs Stem Cell Lineage Specification. Cell 126: 677-689.

ESCUIN S, VERNAY B, SAVERY D, GURNIAK CB, WITKE W, GREENE NDE, COPP AJ (2015). Rho-kinase-dependent actin turnover and actomyosin disassembly are necessary for mouse spinal neural tube closure. J Cell Sci 128: 2468-2481.

GEUSS LR, WU DC, RAMAMOORTHY D, ALFORD CD, SUGGS LJ (2014). Para- magnetic beads and magnetically mediated strain enhance cardiomyogenesis in mouse embryoid bodies. PLoS One 9: 1-20.

GRASHOFF C, HOFFMAN BD, BRENNER MD, ZHOU R, PARSONS M, YANG MT, MCLEAN MA, SLIGAR SG, CHEN CS, HAT, SCHWARTZ MA (2010). Measuring mechanical tension across vinculin reveals regulation of focal adhesion dynamics. Nature 466: 263-266.

HE L, SI G, HUANG J, SAMUEL ADT, PERRIMON N (2018). Mechanical regulation of stem-cell differentiation by the stretch-activated Piezo channel. Nature 555: 103-106.

HEER NC, MARTIN AC (2017). Tension, contraction and tissue morphogenesis. Development 144: 4249-4260.

KRIEG M, DUNN AR, GOODMAN MB (2014). Mechanical control of the sense of touch by $\beta$-spectrin. Nat Cell Biol 16: 224-233.

KUMAR A, OUYANG M, VAN DEN DRIES K, MCGHEE EJ, TANAKAK, ANDERSON MD, GROISMAN A, GOULT BT, ANDERSON KI, SCHWARTZ MA (2016). Talin tension sensor reveals novel features of focal adhesion force transmission and mechanosensitivity. J Cell Biol 213: 371-383.

KUMAR A, PLACONE JK, ENGLER AJ (2017). Understanding the extracellular forces that determine cell fate and maintenance. Development 144: 4261-4270.

LAGENDIJKAK, GOMEZ GA, BAEK S, HESSELSON D, HUGHES WE, PATERSON S, CONWAY DE, BELTING HG, AFFOLTER M, SMITH KA, SCHWARTZ MA, YAP AS, HOGAN BM (2017). Live imaging molecular changes in junctional tension upon VE-cadherin in zebrafish. Nat Commun 8.

MAUL RS, SONG Y, AMANN KJ, GERBIN SC, POLLARD TD, CHANG DD (2003). EPLIN regulates actin dynamics by cross-linking and stabilizing filaments. J Cell Biol 160: 399-407.

MENG F, SACHSF (2011). Visualizing dynamic cytoplasmic forces with a compliancematched FRET sensor. J Cell Sci 124: 261-269.

NIKOLOPOULOU E, GALEA GL, ROLO A, GREENE NDE, COPP AJ (2017). Neural tube closure: cellular, molecular and biomechanical mechanisms. Development 144: 552-566.

NISHIMURA T, HONDA H, TAKEICHI M (2012). Planar cell polarity links axes of spatial dynamics in neural-tube closure. Cell 149: 1084-1097.

ROLOA, SKOGLUND P, KELLERR (2009). Morphogenetic movements driving neural tube closure in Xenopus require myosin IIB. Dev Biol 327: 327-338.

SAWYER JM, HARRELL JR, SHEMER G, SULLIVAN-BROWN J, ROH-JOHNSON M, GOLDSTEIN B (2010). Apical constriction: A cell shape change that can drive morphogenesis. Dev Biol 341: 5-19.

WEST JJ, ZULUETA-COARASAT, MAIER JA, LEE DM, BRUCEAEE, FERNANDEZGONZALEZR, HARRIS TJC (2017). An Actomyosin-Arf-GEF Negative Feedback Loop for Tissue Elongation under Stress. Curr Biol 27: 2260-2270.e5.

YAMASHITAS, TSUBOI T, ISHINABE N, KITAGUCHIT, MICHIUE T (2016). Wide and high resolution tension measurement using FRET in embryo. Sci Rep 6: 28535. 


\section{Further Related Reading, published previously in the Int. J. Dev. Biol.}

Focal adhesion kinase as a mechanotransducer during rapid brain growth of the chick embryo

Mary E. Desmond, Janice E. Knepper, Angela J. DiBenedetto, Elizabeth Malaugh, Sagrario Callejo, Raquel Carretero, Maria-Isabel Alonso and Angel Gato

Int. J. Dev. Biol. (2014) 58: 35-43

Mechanical control of tissue morphogenesis during embryological development

Donald E. Ingber

Int. J. Dev. Biol. (2006) 50: 255-266

Morphomechanics: goals, basic experiments and models

Lev V. Beloussov and Vassily I. Grabovsky

Int. J. Dev. Biol. (2006) 50: 81-92

https://doi.org/10.1387/ijdb.052056lb

Do lamellipodia have the mechanical capacity to drive convergent extension?

G. Wayne Brodland

Int. J. Dev. Biol. (2006) 50: 151-155

https://doi.org/10.1387/ijdb.052040gb

Geometry and mechanics of teleost gastrulation and the formation of primary embryonic axes

Elena M. Cherdantseva and Vladimir G. Cherdantsev

Int. J. Dev. Biol. (2006) 50: 157-168

https://doi.org/10.1387/ijdb.052059ec

The dynamic geometry of mass cell movements in animal morphogenesis

Vladimir G. Cherdantsev

Int. J. Dev. Biol. (2006) 50: 169-182

https://doi.org/10.1387/ijdb.052060vc

Mechanics in embryogenesis and embryonics: prime mover or epiphenomenon?

Richard Gordon

Int. J. Dev. Biol. (2006) 50: 245-253

https://doi.org/10.1387/ijdb.052103rg

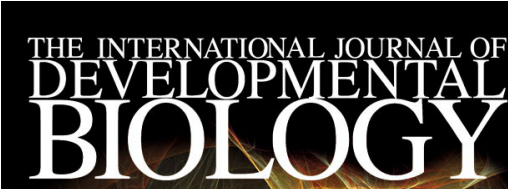

Volume 50 Nos. 2/3 12 sireciah Issue

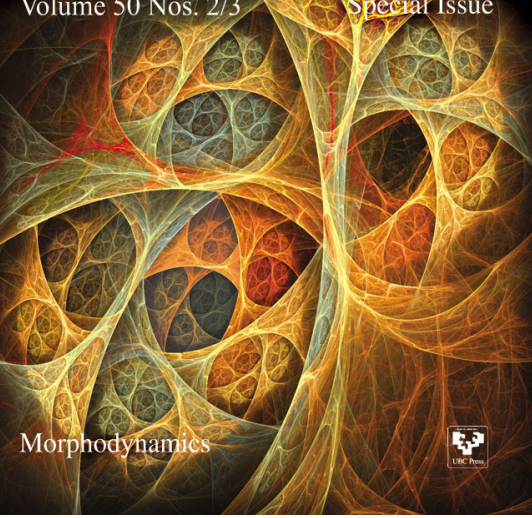

5 yr ISI Impact Factor $(2016)=2.421$
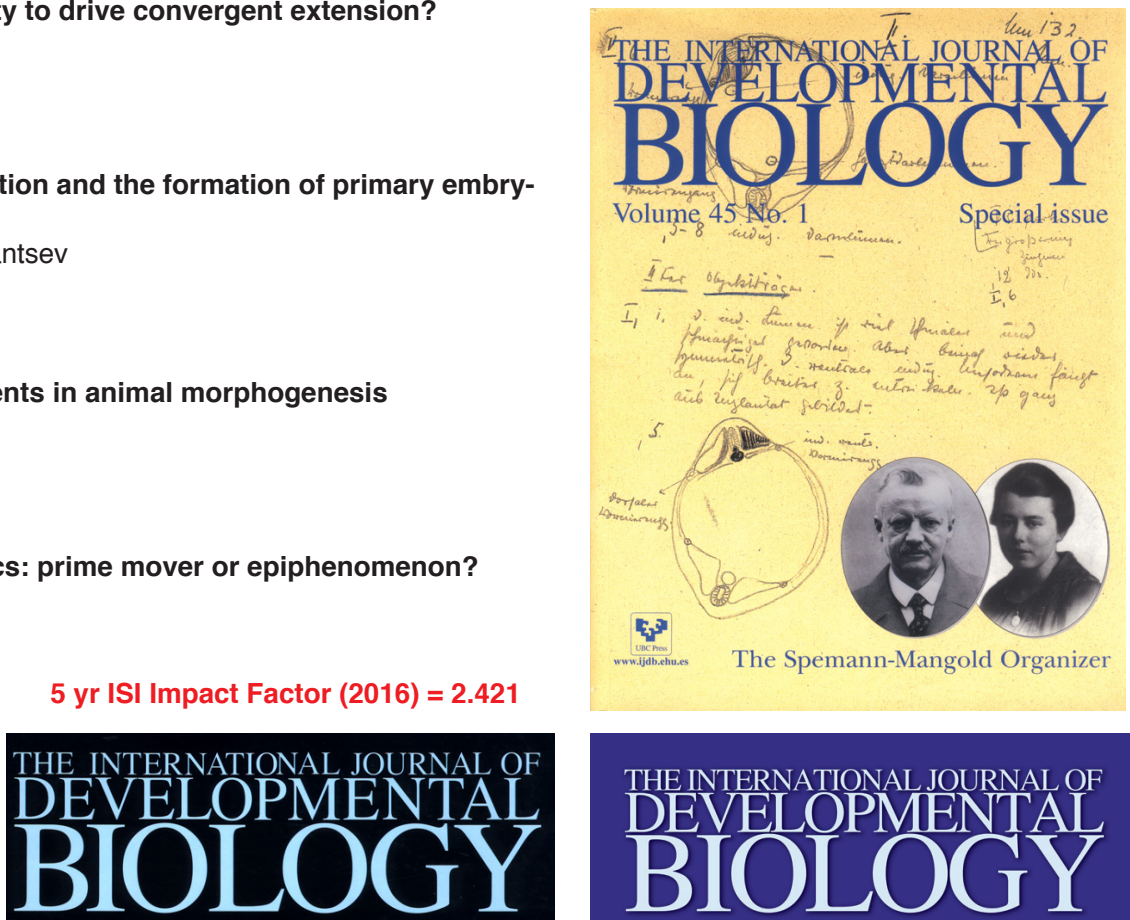
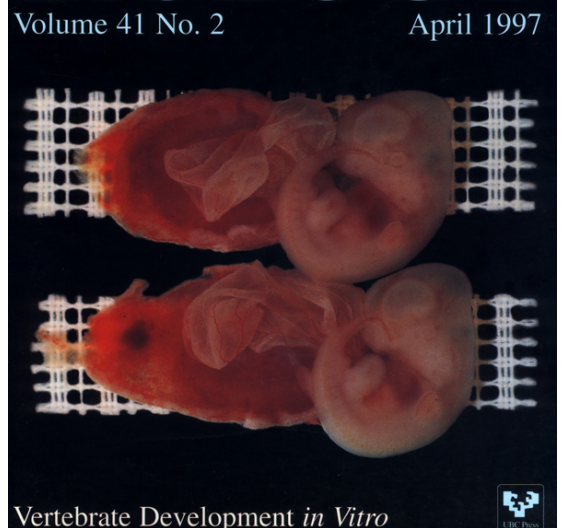

THE INTERNATIONAL JOURNAL OF DEVELOPMIENTAL BUOUU

Volume 62 Nos. 6/7/8

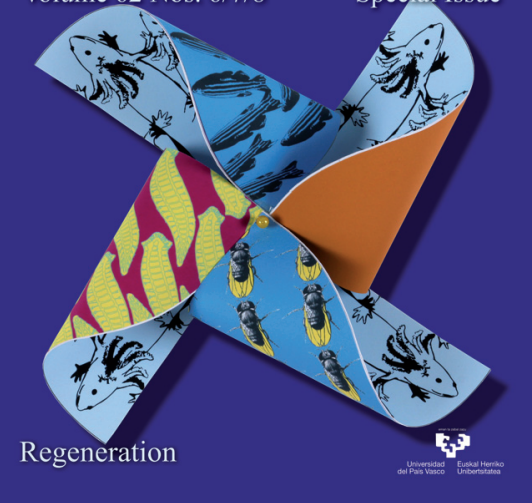

